\title{
IMMUNISATION STATUS OF TWO-YEAR-OLD CHILDREN IN THE HUNTER AREA
}

Thaïs Miles and Chris Wilkinson

Hunter Public Health Unit

Hunter Area Health Service

$\mathrm{T}$ he aim of the investigation described in this article was to estimate the proportion of two-year-old children attending child-care centres in the Hunter Health Area who had received age-appropriate immunisation. Ageappropriate immunisation was defined as having received diphtheria-tetanus-pertussis (DTP 4), sabin (OPV 3) and measles-mumps-rubella (MMR) immunisations within one month of the due date.

There are three types of child-care centres in the Hunter area. One type is home-based child care which is provided by registered people in their own homes. Each person is effectively the director of child care for a very small number of children. A second type is family day care, usually run by local councils. In this case an administrator coordinates the allocation of children to carers and acts as a director of a family day care centre. Such centres cater for a large number of children at different locations. A third type, usually described simply as a child care centre, is organised like a small school with a director and appropriate staff.

The directors of home-based and child-care centres and the administrators of family day care centres are responsible for the maintenance of an up-to-date immunisation register under the 1992 amendment of the Public Health Act 1991. In practice the fulfilment of this requirement is greatly facilitated by frequent contact between the parents and staff members of the centres. However, administrators of family day care are likely to meet with parents only when the children are enrolled into the system. As a result, an unknown number of age-appropriate vaccinations may never be reported to centre staffs or may be recorded in centre registers some considerable time after the immunisations.

\section{METHODS}

Omitting preschools, a list of all 177 child-care centres in the Hunter Health Area was compiled and a 20 per cent random sample of these (comprising 35 centres) was selected. On July 3, 1995 a letter introducing the study was sent to all the centres on the list, not only those sampled. This was partly to provide information to all providers of child care as a courtesy and partly to remind them of their obligation to maintain an up-to-date immunisation register.

A questionnaire was prepared and survey forms were sent to the directors of the listed centres on July 17, 1995, requesting return by August 11, 1995.

The Immunisation Coordinator telephoned centres not responding by the designated date to discuss ways in which the Public Health Unit could facilitate completion of the survey.

\section{RESULTS}

Thirty-three centres responded. Twelve centres reported caring for two-year-old children. Ten of these 12 centres provided relevant information on a total of 226 children. The other two centres did not respond despite follow-up telephone calls.

Information on whether the children were Aboriginal or Torres Strait Islanders was omitted in only four cases. None of the remaining 222 children was identified as Aboriginal or Torres Strait Islander.

Data on Haemophilus influenzae type b (Hib) immunisation followed no particular pattern. This may have been due to changes in Hib vaccine pricing policy in July and September 1993. Consequently the Hib vaccination rate was not investigated.

The proportions of the 226 children found to have received age-appropriate immunisation were:

$\begin{array}{ll}\text { DTP 4 } & 43 \% \\ \text { OPV 3 } & 67 \% \\ \text { MMR } & 58 \%\end{array}$

These rates were based on the responses pertaining to 171 family day care children and 69 others. Rates for family day care children alone were very low compared with the rates for the remaining children. This was attributed to a low level of immunisation reporting by parents to family day care centre staff. It was therefore decided to calculate the required proportions from the data provided by child-care centres other than family day care centres.

The proportions of the remaining 69 children found to have received age-appropriate immunisation were:

$\begin{array}{ll}\text { DTP 4 } & 77 \% \\ \text { OPV 3 } & 83 \% \\ \text { MMR } & 83 \%\end{array}$

\section{DISCUSSION}

The total number of two-year-old children in the Hunter Area is about 7,500. Extrapolation of the findings of this study to all Hunter two-year-old children is not possible. There is no available information on the immunisation status of two-year-old children who do not attend child care. Two sets of estimates are provided by this survey. The first describes the immunisation status of most of the sampled children but is considered to be a gross under-estimate. The second set of estimates is considered more likely to represent the true immunisation status of Hunter children in child care.

In view of the questionable validity of data from family day care centres and the difficulty of carrying out a related study which had to be abandoned, it is recommended that the NSW Health Department works co-operatively with family day care administration to improve the procedures for registering vaccinations. Discussion of possible changes could include the assumption by the carer of the responsibility for obtaining and recording immunisation data and transmitting it to the administrator.

It is also suggested that the set of estimates based on the sample which excluded family day care children be regarded as the best indices available at present for Hunter children.

The Immunisation Program Coordinator, NSW Health Department, has recently had discussions with the president of the Family Day Care Association in an effort to identify ways of assisting family day care personnel to comply with the immunisation provisions of the Public Health Act 1991. The Coordinator will address the association's February 1997 meeting on this matter.

- EDITOR 